\title{
Dicer 1, ribonuclease type III modulates a reprogramming effect in colorectal cancer cells
}

\author{
DYAH LAKSMI DEWI ${ }^{1}$, HIDESHI ISHII ${ }^{2}$, NAOTSUGU HARAGUCHI ${ }^{2}$, SHIMPEI NISHIKAWA $^{1}$, \\ YOSHIHIRO KANO ${ }^{1}$, TAKAHITO FUKUSUMI ${ }^{1}$, MIYUKI OZAKI ${ }^{2}$, TOSHIYUKI SAITO ${ }^{3}$, \\ DAISUKE SAKAI ${ }^{2}$, TAROH SATOH $^{2}$, YUICHIRO DOKI $^{1}$ and MASAKI MORI ${ }^{1}$
}

\begin{abstract}
Departments of ${ }^{1}$ Gastroenterological Surgery and ${ }^{2}$ Frontier Science for Cancer and Chemotherapy, Osaka University Graduate School of Medicine, Osaka 565-0871; ${ }^{3}$ Transcriptome Profiling Group, National Institute of Radiological Sciences, Research Center for Charged Particle Therapy, Chiba 263-8555, Japan
\end{abstract}

Received December 16, 2011; Accepted February 2, 2012

DOI: $10.3892 / \mathrm{ijmm} .2012 .945$

\begin{abstract}
Complete cell reprogramming can be achieved by the introduction of specific transcription factors, Oct4 [also known as POU class 5 homeobox 1 (Pou5f1)]; sex-determining region Y (SRY)-box 2 (Sox2); Kruppel-like factor 4 (Klf4); and myelocytomatosis viral oncogene homolog (c-Myc), into terminally differentiated mouse somatic fibroblasts. This reprogramming process may be accelerated or suppressed by various factors, including microRNAs (miRNAs). Introduction of these transcription factors or miRNAs considerably modifies the malignant phenotype of cancer cells. We studied the effect of introducing these transcription factors into two distinct colorectal cancer (CRC) cell lines, HCT116 and DLD-1, in the presence and absence of Dicer 1, ribonuclease type III (Dicer1), a critical miRNA processing enzyme. We assessed cell reprogramming based on the number of cells exhibiting alkaline phosphatase staining and an increase in embryonic stem cell-like gene expression, indicating the return of cells to an immature state. Dicerl-deficient CRC cells showed a reduced number of alkaline phosphatase-positive reprogrammed cells than wild-type (WT) cells. Before reprogramming, endogenous expression of an immature carbohydrate epitope, TRA-1-60, was high in Dicer1-deficient CRC cells, whereas after reprogramming, the expression of this epitope was increased in Dicer1-sufficient more than in Dicer1-deficient CRC cells. Our data demonstrate the critical
\end{abstract}

Correspondence to: Dr Masaki Mori, Department of Gastroenterological Surgery, Osaka University Graduate School of Medicine, Yamadaoka 2-2, Suita, Osaka 565-0871, Japan

E-mail: mmori@gesurg.med.osaka-u.ac.jp

Dr Hideshi Ishii, Department of Frontier Science for Cancer and Chemotherapy, Osaka University Graduate School of Medicine, Yamadaoka 2-2, Suita, Osaka 565-0871, Japan

E-mail: hishii@cfs.med.osaka-u.ac.jp

Key words: Dicer 1, ribonuclease type III, cancer, colon, rectum, reprogramming, microRNA role of miRNAs in the reprogramming process and determination of a differentiated phenotype of CRC cells.

\section{Introduction}

Complete cell reprogramming can be achieved by the introduction of specific transcription factors, Oct4 [also known as POU class 5 homeobox 1 (Pou5f1)]; sex-determining region $\mathrm{Y}$ (SRY)-box 2 (Sox2); Kruppel-like factor 4 (Klf4); and myelocytomatosis viral oncogene homolog (c-Myc), into terminally differentiated somatic fibroblasts of mice (1) and humans (2); somatic fibroblasts reprogrammed in this manner are termed induced pluripotent stem (iPS) cells. This reprogramming process can be enhanced by co-expression of microRNAs (miRNAs), a subclass of non-coding RNAs, such as miR-17 (3), miR-93 (3), miR-106s (3), miR-200c (4), miR-294 (5), miR302s (4-6), miR-367 (6), miR-369s (4), and miR-372 (5). Even in the absence of the specific transcription factors, the introduction of miR-200c, miR-302s, and miR-369s is sufficient to induce a pluripotent state (4). Thus, it is apparent that the miR network plays a critical role in the regulation of the reprogramming process in normal differentiated cells. This discovery is of particular significance for regenerative medicine using non-cancerous cells.

We recently reported that the introduction of these transcription factors into gastrointestinal cancer cells resulted in their acquisition of a multidifferentiation potential, i.e., the gene expression profiles of mesoderm and ectoderm appeared in the gastrointestinal cancer cells of endodermal origin [iPSlike cancer (iPC) cells] (7). Reprogramming of sensitized cancer cells in response to differentiation therapy suppressed tumorigenicity in vivo (7); this presumably involved reactivation of tumor suppressor genes at the cyclin-dependent kinase inhibitor $C D K N 2 b-C D K N 2 a$ locus on chromosome 9p21 in humans (chromosome 4 in mice), a region that is frequently inactivated in cancerous cells and is involved in the reduction of chemosensitivity (8). Furthermore, a study on melanoma (conducted using Colo-16 keratinocytes) and prostate cancer (conducted using the PC3 cell line) showed that the introduction of miR-302s resulted in a reduction in the migratory ability 
and in the expression of the cell cycle-related genes, cyclin D1, cyclin D2, and cyclin-dependent kinase 2; DNA methylation facilitators, methyl-CpG-binding protein (MECP)2 and MECP1-p66; and melanoma oncogenes (9). These findings strongly suggest that epigenetic modifications achieved using iPS technology with specific transcription factors and miRNAs may be sufficient to reverse the differentiation program and lead to a multipotent state while also contributing to the suppression of biologically malignant phenotypes of cancer cells.

Here we study the effects of the introduction of specific iPS transcription factors into colorectal cancer (CRC) cells in both the presence and absence of the miRNA processing enzyme Dicer 1, ribonuclease type III (Dicer1). Our data show the critical role of miRNAs in the reprogramming process and determination of a differentiated phenotype of CRC cells.

\section{Materials and methods}

Cell culture. Two pairs of cell lines, wild-type (WT) and Dicer1-deficient CRC cell lines, were donated by Bert Vogelstein (Johns Hopkins University, Baltimore, MD, USA): i) WT HCT116 cells and their homologous-recombination mutant form, Dicer1-deficient HCT116 cells and ii) WT DLD-1 cells and their mutant form, Dicer1-deficient DLD-1 cells (10). The cell lines were maintained in Dulbecco's modified Eagle's medium (DMEM; Nacalai Tesque, Kyoto, Japan) supplemented with $10 \%$ fetal bovine serum (FBS) at $37^{\circ} \mathrm{C}$ in a $5 \%$ humidified $\mathrm{CO}_{2}$ atmosphere. Gene transfer of specific transcription factors, Oct4, Sox 2, Klf4 and c-Myc, was performed using lentiviral and retroviral vector systems as previously described (7). The plasmids of these specific transcription factors were purchased from Addgene (Cambridge, MA, USA). In brief, the cancer cell lines were transfected with the plasmids at a concentration of $4 \mu \mathrm{g} / \mu \mathrm{l}$ using Lipofectamine 2000 (Invitrogen, Carlsbad, CA, USA) and incubated in glucosefree Opti-MEM (Invitrogen). All retroviral transfections were performed using the ViraDuctin Retrovirus Transduction kit (Cell Biolabs, San Diego, CA, USA). Lentiviral transfections were performed using ViraPower Lentiviral Packaging mix (Invitrogen). Transfectants were grown in DMEM supplemented with $10 \%$ FBS and puromycin $(2 \mu \mathrm{g} / \mathrm{ml})$. The Nanog (a homeobox transcription factor) promoter-driven green fluorescent protein (GFP) was used as an indicator of plasmid transfection (7). An all-in-one-type fluorescence microscope (BZ-8000; Keyence, Osaka, Japan) with digital photographic capability was used to visualize cells at several magnifications. For immunocytology, we used antibodies against TRA-1-60 (Chemicon International, Inc., Temecula, CA, USA). Alkaline phosphatase (AP) staining was performed using the staining kit (MBL, Nagoya, Japan) to test the induction of iPS-like cancer cells (iPC) as described previously $(1,2)$. The colonies were stained by crystal violet.

$R N A$. Total-RNA was prepared using the TRIzol reagent (Invitrogen). Reverse transcription (RT) was performed using SuperScript III Reverse Transcriptase kits (Invitrogen). To confirm polymerase chain reaction (PCR) amplification, 25-35 cycles of PCR were performed using the PCR amplification kit (Takara Bio, Inc., Kyoto, Japan) and the GeneAmp PCR System 9600 (PE Applied Biosystems, Foster City, CA, USA) under the following conditions: $95^{\circ} \mathrm{C}$ for $10 \mathrm{sec}, 60^{\circ} \mathrm{C}$ for $10 \mathrm{sec}$, and $72^{\circ} \mathrm{C}$ for $60 \mathrm{sec}$. An $8-\mu 1$ aliquot of each reaction mixture was size-fractionated on a $1.5 \%$ agarose gel and visualized by ethidium bromide staining. For quantitative assessment, gene expression was evaluated by quantitative real-time PCR (qRT-PCR) using a LightCycler TaqMan Master kit (Roche Diagnostics, Tokyo, Japan) for cDNA amplification of specific target genes (7).

Statistical analysis. For continuous variables, our results are expressed as means \pm standard errors. The relationship between the gene expression level and cell count was analyzed using Chi-square test and Wilcoxon signed rank and rank sum tests. All data were analyzed using the JMP software (SAS Institute, Cary, NC, USA). Differences with $\mathrm{P}<0.05$ were considered statistically significant.

\section{Results and Discussion}

Introduction of transcription factors. Given that the introduction of specific transcription factors induces reprogramming of both normal differentiated cells $(1,2)$ and cancer cells $(7)$, we performed lentiviral and retroviral transfection of these transcription factors, Oct4, Sox2, Klf4 and c-Myc, on Day 0. The cells were plated on mouse embryonic fibroblasts (MEFs), and AP staining and TRA-1-60 scoring were performed on Days 12 and 28, respectively. On Day 30, colonies were picked and characterized as per the schedule shown in Fig. 1A. Transfection of WT HCT116, mutant Dicer1-deficient HCT116, WT DLD-1, and mutant Dicer1-deficient DLD-1 cells (Fig. 1B) resulted in the formation of iPC colonies on MEFs (Fig. 1C). The colonies showed a round-shaped phenotype, and cells were concentrated and their intracellular membranes were invisible; all these characteristics are reminiscent of iPS cells.

Expression of embryonic stem cell (ESC)-like genes. Next, we assessed expression of ESC-like genes. qRT-PCR indicated that the expression of Oct 4 and Sox 2 increased 30 days after transfection (Fig. 2), while the expression of transgenes was downregulated presumably because of transgene silencing (data not shown) $(1,2)$. Expression of Nanog, which was not considered a transgene, increased after transfection of the transcription factors (Fig. 2).

Alkaline phosphatase staining. To characterize the immature cell states, we performed AP staining of iPC cells $(1,2)$. Data indicated that the frequency of AP staining was high in WT vs. Dicer-deficient HCT116 and DLD-1 cells (HCT116: 310 vs. 220 out of $1 \times 10^{6}$ cells; DLD-1: 80 vs. 15 out of $1 \times 10^{6}$ cells) (Fig. 3). The results indicate the requirement for Dicerl in the reprogramming of HCT116 and DLD-1 human CRC cells.

TRA-1-60 expression. To further characterize immature cell states, we performed TRA-1-60 staining of iPC cells $(1,2)$. Before reprogramming, TRA-1-60 expression was high $(8.8 \%)$ in Dicer1-deficient HCT116 cells compared to that in WT HCT116 cells (0.6\%; Fig. 4A and B), suggesting that the absence of Dicer1, i.e., the amount of miRNA, may influence the cellular differentiation program. Interestingly, 30 days after transfection of the transcription factors, the population 
A

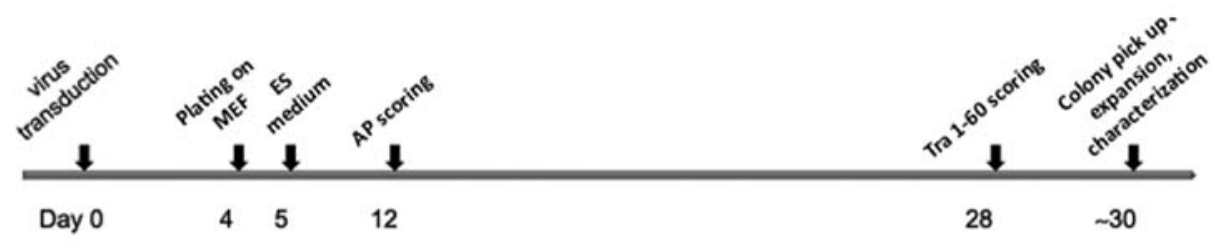

B

C

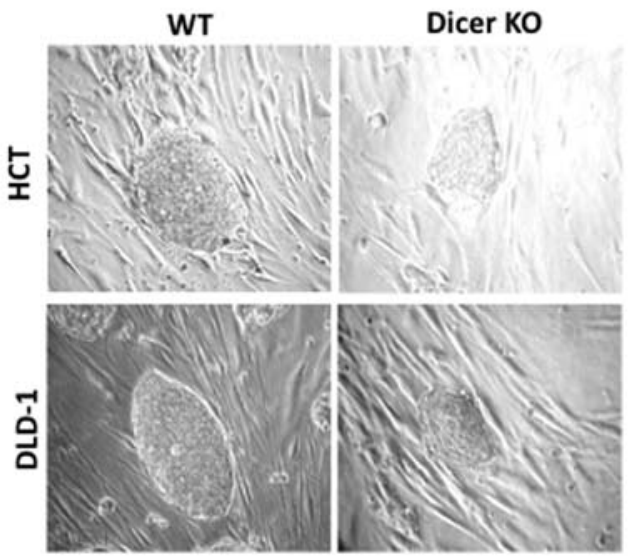

Figure 1. Retroviral- and lentiviral-mediated gene transfer of four iPS factor genes in CRC cells. (A) Schematic representation of the experiment. (B) Genotyping PCR for wild-type (WT) and Dicer1-deficient (DKO) HCT116 and DLD-1 cells performed as previously described (10). (C) Morphological changes in iPC cells derived from WT and Dicer1-deficient HCT116 and DLD-1 cells by phase contrast microscopy.
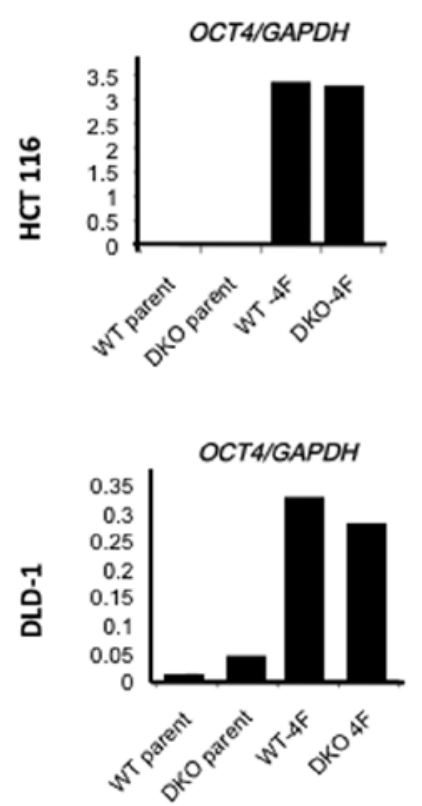
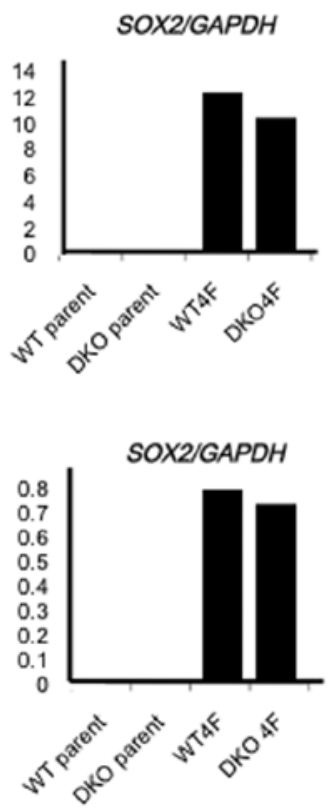
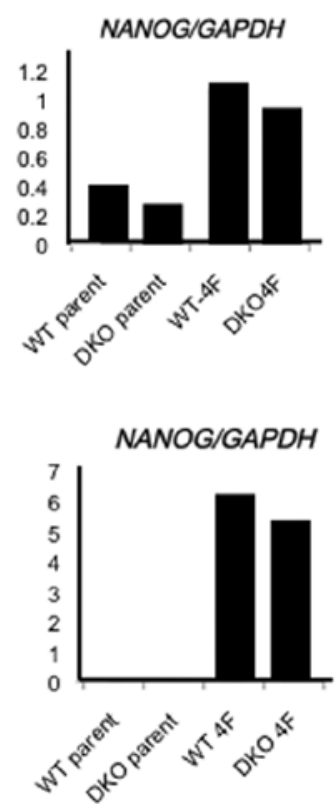

Figure 2. Exogenous and endogenous gene expression by iPS cells after retroviral- and lentiviral-mediated gene transfer. After viral vector-mediated gene transfer of the four defined transcription factor genes, c-Myc, Sox2, Oct4 and K1f4, expression of embryonic stem cell-like genes Oct4, Sox 2 and Nanog was assessed in wild-type (WT 4F) and Dicerl-deficient (DKO 4F) in HCT116 and DLD-1 cells. The graphs represent levels measured by qRT-PCR (the relative levels of expression are shown in arbitrary units).

of TRA-1-60-positive cells increased approximately 7.2-fold (from 0.6 to $4.3 \%$ ) in WT HCT116 cells, whereas this increase was only $\sim 1.1$-fold (from 8.8 to $10.0 \%$; Fig. 4A-C) in Dicer1deficient HCT116 cells. These data indicate that Dicer1 plays a critical role in CRC cell reprogramming and strongly suggest the necessity of microRNAs in this reprogramming process.

Cancer cells generally harbor genetic instability, and the extent to which epigenetic modifications associated with repro- gramming modulate the biological behavior of tumor cells remains debatable. Previous studies indicated that transfection of specific transcription factors (7) or miR-302s (9) resulted in a considerable modulation of the malignant phenotypes of cancer cells, i.e., invasion, cell growth, tumorigenicity, and sensitivity to chemotherapeutic reagents and differentiation reagents, such as vitamins. miRNAs modulate mRNA expression through base pairing between seed sequences in miRNAs 

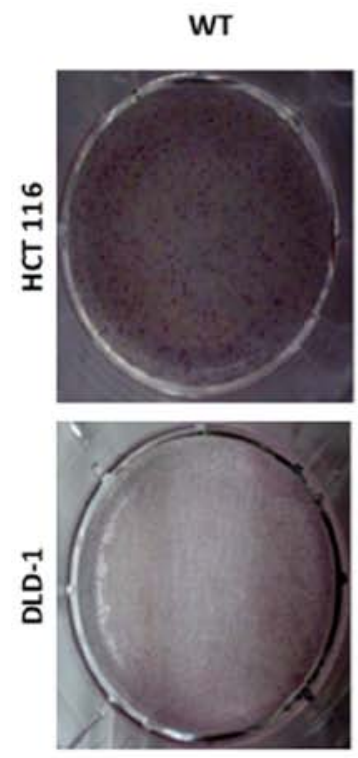

Dicer KO

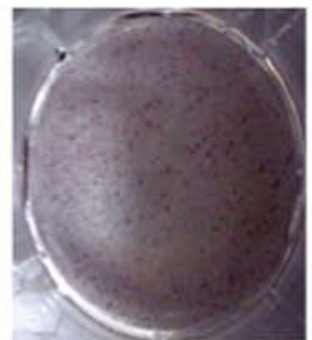

WT
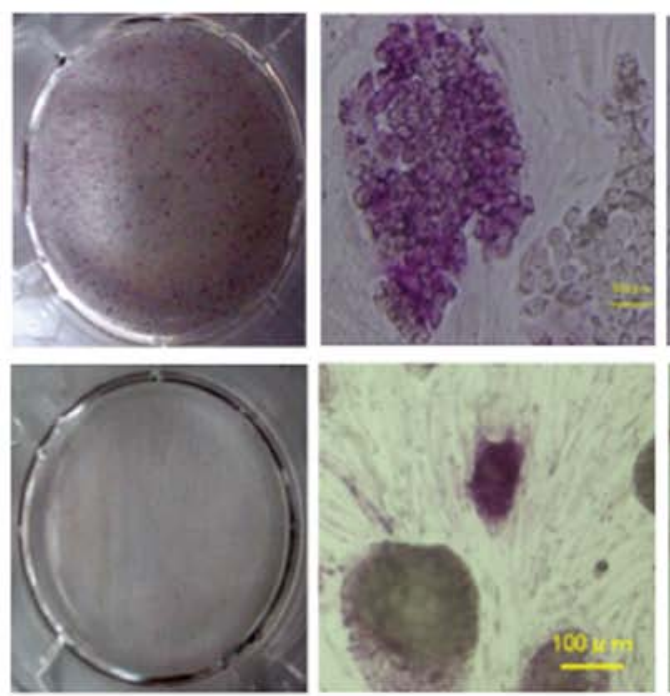

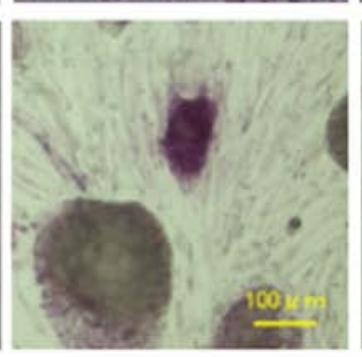

Dicer KO
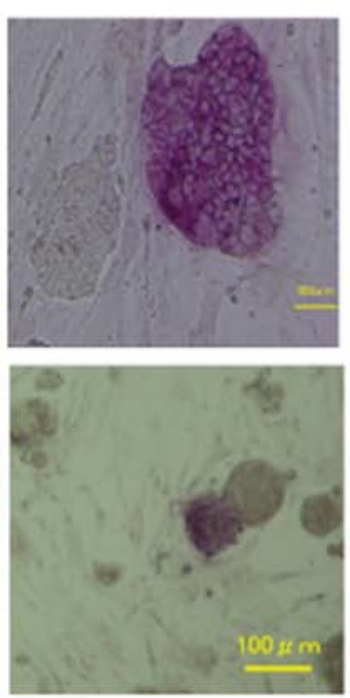

HCT116

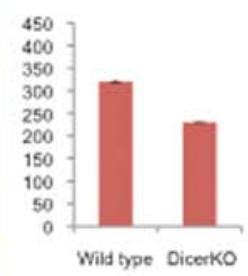

DLD1

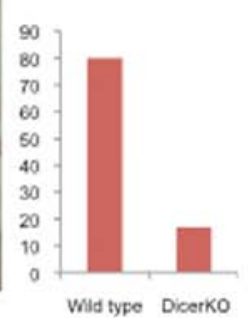

Figure 3. Alkaline phosphatase staining of iPC cells. The reprogramming of iPC cells from WT and Dicer1-deficient (HCT116 and DLD-1) cells was evaluated by AP staining. Left, 10-cm dishes; middle, colony morphology; right, number of colonies is presented on the Y-axis.

A
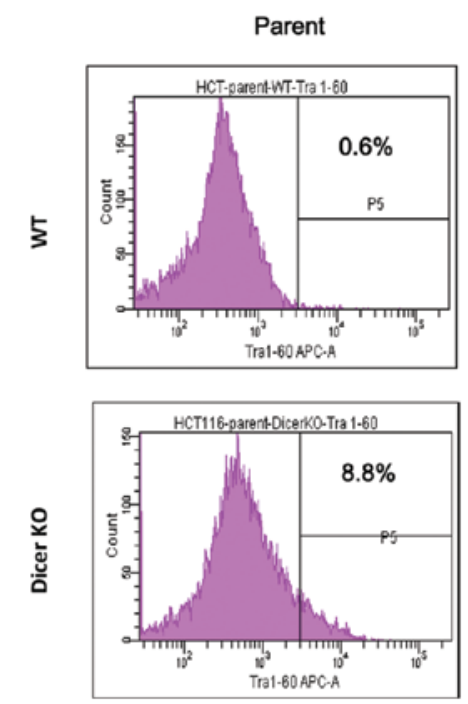

B
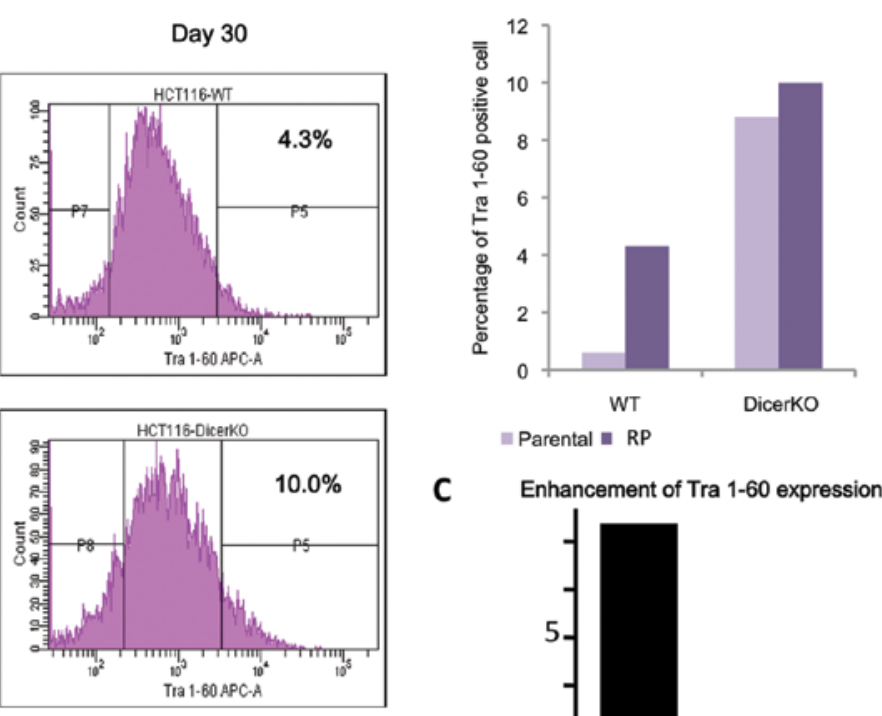

Day 30

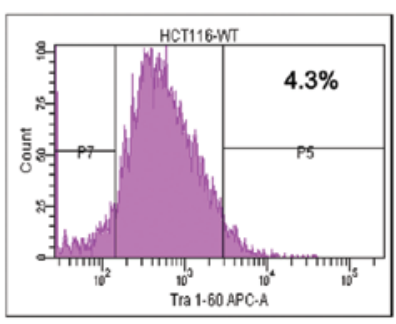

C

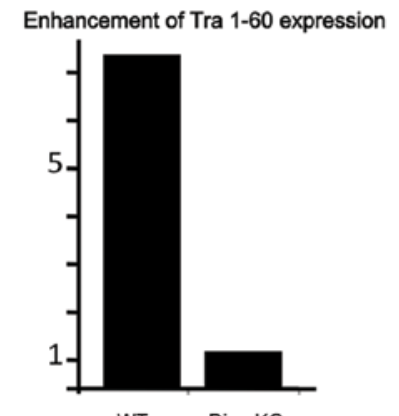

WT Dicerko

Figure 4. Efficiency of reprogramming induced by specific transcription factors. (A) Flow cytometry shows the population of TRA-1-60-positive cells (horizontal axis) on Day 30. (B) The percentage of TRA-1-60-positive cells. (C) The increased ratio of TRA-1-60-positive cells in WT relative to those in Dicer KO. WT, wild-type cells; KO, knockout deficient cells.

and complementary sequences within the open reading frame or untranslated regions of the target mRNA, thereby destabilizing mRNA and/or inhibiting protein synthesis $(11,12)$. Moreover, miRNAs play crucial roles in developmental processes $(13,14)$, stress response $(15,16)$, stem cell physiology $(13,14,17)$, and diseases such as cancer $(10,18-21)$. The present study indicates that the introduction of defined reprogramming transcription factors, such as Oct4, Sox2, Klf4 and c-Myc, results in reversion of CRC cells to an iPS-like phenotype, which is more prominent in the presence, than in the absence, of Dicerl, an enzyme critical for miRNA maturation.

In conclusion, our data indicate that miRNA expression is necessary for cancer cell reprogramming and suggest that the introduction of several miRNAs modulates or enhances this reprogramming effect not only in normal but also in cancer cells and may thus be applied in future therapies. 


\section{Acknowledgements}

We thank Dr Bert Vogelstein (Johns Hopkins University, Baltimore, MD, USA) for providing Dicerl-deficient cell lines. This study was partly supported by a grant from the Core Research for Evolutional Science and Technology (CREST) (M.M., H.I., N.H.), a Grant-in-Aid for Scientific Research on Priority Areas (M.M.), a Grant-in-Aid for Scientific Research from the Ministry of Education, Culture, Sports, Science and Technology (M.M., H.I., N.H.), a Grant-in-Aid for the Third Comprehensive 10-year Strategy for Cancer Control Ministry of Health, Labour and Welfare (M.M., H.I.), a grant from the Tokyo Biochemical Research Foundation (M.M.), and a grant from the Princess Takamatsu Cancer Research Fund, Japan (H.I.).

\section{References}

1. Takahashi $\mathrm{K}$ and Yamanaka S: Induction of pluripotent stem cells from mouse embryonic and adult fibroblast cultures by defined factors. Cell 126: 663-676, 2006.

2. Takahashi K, Tanabe K, Ohnuki M, Narita M, Ichisaka T, Tomoda K and Yamanaka S: Induction of pluripotent stem cells from adult human fibroblasts by defined factors. Cell 131: 861-872, 2007

3. LiZ, Yang CS, Nakashima K and Rana TM: Small RNA-mediated regulation of iPS cell generation. EMBO J 30: 823-834, 2011.

4. Miyoshi N, Ishii H, Nagano H, Haraguchi N, Dewi DL, Kano Y, Nishikawa S, Tanemura M, Mimori K, Tanaka F, et al: Reprogramming of mouse and human cells to pluripotency using mature microRNAs. Cell Stem Cell 8: 633-638, 2011.

5. Subramanyam D, Lamouille S, Judson RL, Liu JY, Bucay N, Derynck R and Blelloch R: Multiple targets of miR-302 and miR-372 promote reprogramming of human fibroblasts to induced pluripotent stem cells. Nat Biotechnol 29: 443-448, 2011.

6. Anokye-Danso F, Trivedi CM, Juhr D, Gupta M, Cui Z, Tian Y, Zhang Y, Yang W, Gruber PJ, Epstein JA and Morrisey EE: Highly efficient miRNA-mediated reprogramming of mouse and human somatic cells to pluripotency. Cell Stem Cell 8: 376-388, 2011.
7. Miyoshi N, Ishii H, Nagai K, Hoshino H, Mimori K, Tanaka F, Nagano H, Sekimoto M, Doki Y and Mori M: Defined factors induce reprogramming of gastrointestinal cancer cells. Proc Natl Acad Sci USA 107: 40-45, 2010.

8. Gil J and Peters G: Regulation of the INK4b-ARF-INK4a tumour suppressor locus: all for one or one for all (Review). Nat Rev Mol Cell Biol 7: 667-677, 2006.

9. Lin SL, Chang DC, Chang-Lin S, Lin CH, Wu DT, Chen DT and Ying SY: Mir-302 reprograms human skin cancer cells into a pluripotent ES-cell-like state. RNA 14: 2115-2124, 2008.

10. Cummins JM, He Y, Leary RJ, Pagliarini R, Diaz LA Jr, Sjoblom T, Barad O, Bentwich Z, Szafranska AE, Labourier E, et al: The colorectal microRNAome. Proc Natl Acad Sci USA 103: 3687-3692, 2006.

11. Ambros V: The functions of animal microRNAs. Nature 431 350-355, 2004.

12. John B, Enright AJ, Aravin A, Tuschl T, Sander C and Marks DS: Human microRNA targets. PLoS Biol 2: e363, 2004.

13. Houbaviy HB, Murray MF and Sharp PA: Embryonic stem cellspecific microRNAs. Dev Cell 5: 351-358, 2003.

14. Lim LP, Glasner ME, Yekta S, Burge CB and Bartel DP: Vertebrate microRNA genes. Science 299: 1540, 2003.

15. Leung AK and Sharp PA: MicroRNA functions in stress responses. Mol Cell 40: 205-215, 2010.

16. Marsit CJ, Eddy K and Kelsey KT: MicroRNA responses to cellular stress. Cancer Res 66: 10843-10848, 2006.

17. $\mathrm{Ng} \mathrm{HH}$ and Surani MA: The transcriptional and signalling networks of pluripotency. Nat Cell Biol 13: 490-496, 2011.

18. Calin GA, Dumitru CD, Shimizu M, Bichi R, Zupo S, Noch E, Aldler H, Rattan S, Keating M, Rai K, et al: Frequent deletions and down-regulation of micro-RNA genes miR15 and miR16 at $13 q 14$ in chronic lymphocy tic leukemia. Proc Natl Acad Sci USA 99: 15524-15529, 2002.

19. Calin GA, Ferracin M, Cimmino A, Di Leva G, Shimizu M, Wojcik SE, Iorio MV, Visone R, Sever NI, Fabbri M, et al: A microRNA signature associated with prognosis and progression in chronic lymphocytic leukemia. N Engl J Med 353: 1793-1801, 2005.

20. Cerchia L and De Franciscis V: Noncoding RNAs in Cancer Medicine. J Biomed Biotechnol 2006: 73104, 2006.

21. Wijnhoven B, Michael MZ and Watson DI: MicroRNAs and cancer (Review). Br J Surg 94: 23-30, 2007. 